\title{
Study of the aerodynamic structure around a NACA6409 Aairfoil rotor
}

\author{
Saoussane Gouiaa, Ameni Mehdi, Zied Driss, Bilel Ben Amira, Mohamed Salah Abid \\ Laboratory of Electro-Mechanic Systems (LASEM), National School of Engineers of Sfax (ENIS), University of Sfax (US), \\ B.P. 1173, Road Soukra km 3.5, 3038 Sfax, TUNISIA \\ guina@gmail.com
}

\begin{abstract}
The multi-copters are one of the important aircrafts in the world because they are used in both military and civil application. The problem of these aircrafts is the low efficiency of its propellers. That's why, it is necessary to study the aerodynamic structure around every rotor. In this paper, a computer simulation has been done to study the aerodynamic structure around a NACA 6409 airfoil rotor. The numerical model is based on the resolution of the Navier - Stockes equations with a standard $k-\varepsilon$ model. Then, the finite volume discretization of these equations leads to solve it. The local characteristics of the aerodynamic structure are extracted using the software Solidworks Flow Simulation and the validation is done with comparison with anterior result of Alexandrov [1].
\end{abstract}

Keywords: NACA 6409 airfoil rotor, aerodynamic structure, computer simulation, CFD.

Received: 12/02/2017 - Accepted: 11/04/2017

\section{Introduction}

The study of the aerodynamic structure around a propeller has an important value not only in academic research but also in some industries. In fact, these types of propellers are used especially in multi-copters which are a specific category of UAVs. These machines are used in military and civil applications. That's why, we can deduce their importance. So, it is interesting to solve every problem related to the propellers which are called also rotors. In fact, due to the low efficiency of the rotors, the academic researchers discovered new technologies to decrease the consumption of energy. In this context, they investigated numerical models and experiments to improve their performances. In fact, the calculated the value of the thrust force and determined the local characteristics of the aerodynamic structure. For example, Alexandrov [1] determined the thrust force of one rotor using CFD and experiments. He used the same method also to choose the best configuration of propellers that saves the maximum of energy. Also, Le Pape et al. [2] investigated a procedure that leads to the optimization of the helicopter rotor aerodynamic performance. In fact, the optimizer has been coupled to a 3D Navier-Stockes CFD solver and applied to helicopter rotor optimization in hover.

verification. Indeed, they used the CFD as a prediction for the viscous, compressible flow-field in the tip region and
Heyong et al. [3] simulated the unsteady flows around forward flight helicopter with coaxial rotors based on unstructured dynamic overset grids. The performances of the two coaxial rotors both become worse because of the aerodynamic interaction between them, and the interaction between the two rotors. When the spacing between the two coaxial rotors increases, the thrust of the top rotor increases and the total thrust coefficient is reduced. Zhao et al. [4] studied the CFD analysis of a ducted-Fan UAV based on Magnus effect. In this vehicle, the actuator system consists of four rotary cylinders which are symmetrically installed at bottom of inside duct. The force used for attitude stabilization is generated by the interaction between the surface of cylinder and the downwash, which is known as Magnus effect. In this paper, the aerodynamic characteristics of propeller-wing interaction for the ducted fan UAV were simulated numerically based on the Computational Fluid Dynamics (CFD) by means of sliding mesh technology. Brocklehurst et al. [5] evaluated the performance of a new tip design of helicopter rotor blade. They investigated new rotor designs which are still a subject of intensive flight test

thus a prediction of the performance of the blade. From the previous studies, it appears that it is important to 
investigate new designs.

In this paper, we are interested on the study of the NACA 6409 airfoil rotor. , configurations or profiles of propellers to increase their performance.

\section{Geometrical arrangement}

Figure 1 presents the geometrical model. It is composed of a 10x5 propeller having a NACA 6409 airfoil and mounted in a brushless motor. The value $D=10$ inch presents the diameter of the propeller and 5 presents the value of the pitch in inch also. The used model is similar to the one used by Alexandrov [1].

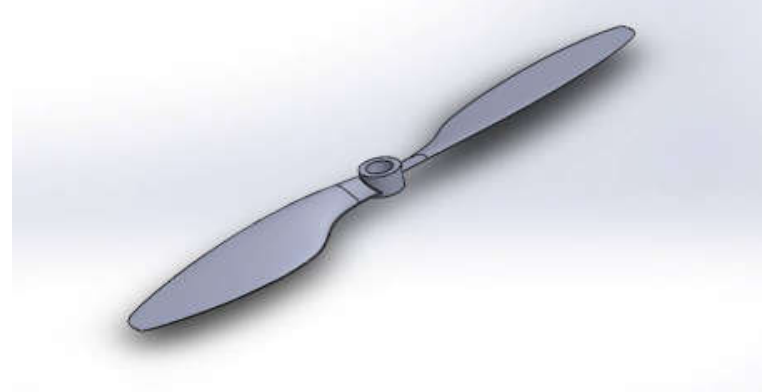

(a) NACA 6409 airfoil (10x5 propeller)

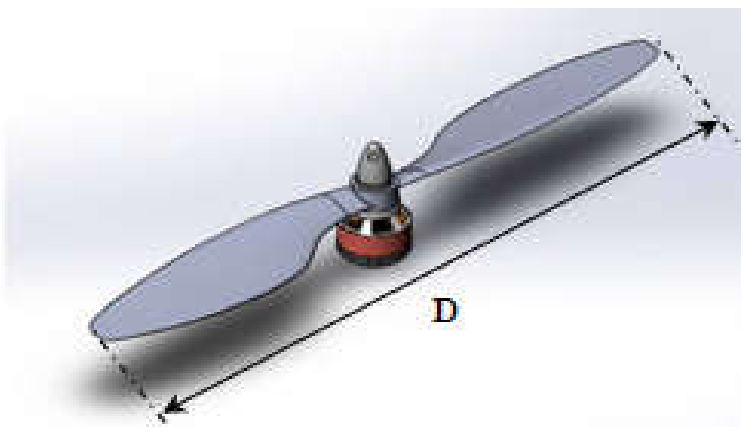

(b) Studied geometry (propeller mounted in a brushless motor)

Figure 1. Geometrical model

\section{Numerical model}

To study the local characteristics of the aerodynamic structure around a NACA 6409 airfoil rotor, we have used the software "Solidworks Flow Simulation". "Solidworks Flow Simulation" is a class of Computational Fluid Dynamics (CFD) analysis software that is fully embedded in the mechanical design environment, for all engineering applications. The "Solidworks Flow Simulation" incorporates a number of technologies like CAD data management, mesh generation, CFD solvers, engineering modeling technologies and result processing [6]. Flow simulation is capable to detect both laminar and turbulent flows. Laminar flow is determined when the Reynolds number, which is a dimensionless number that gives a measure of the ratio of inertial forces to viscous forces, is low. Over a critical value of Reynolds number, the flow becomes turbulent. The numerical model is based on the resolution of Navier-Stokes equations with a standard k- $\varepsilon$ model [7]. "Solidworks Flow Simulation" calculation was performed in a rectangular parallelepiped-shaped computational domain. The boundary conditions are orthogonal to the axes of the global coordinate system. A computational mesh vises the computational domain with a set of plane orthogonal to the coordinate system's axes to form rectangular parallelepipeds called cells. The resulting computational mesh consists of cells of different types. These cells can be divided into triangles. After using Solidworks to create the model, we use "Flow Simulation" to simulate the fluid environment of the object and the effects that can affect the model according to the following design cycle shown in figure 2 [8-9]. To simulate the model, it was important to choose the adequate boundary conditions. In the present application, the pressure is equal to $101325 \mathrm{~Pa}$ and the air molecular mass is equal to $0.02896 \mathrm{~kg} / \mathrm{mol}$. The adequate numerical model is the one that gives a curve of the force of thrust in function of the rotational speed near to the experimental curve of the experimental results of Alexandrov [1].

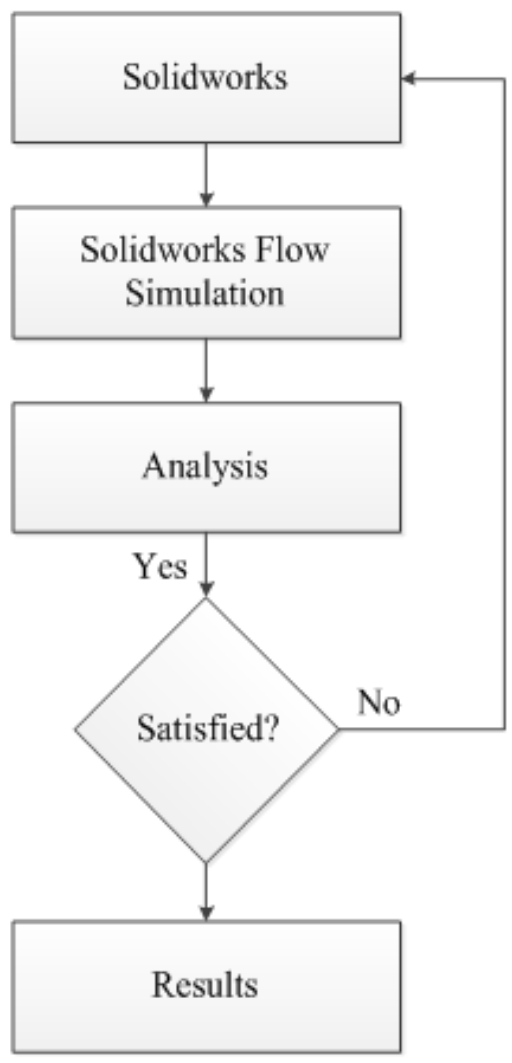

Figure 2. Solidworks' procedure 


\section{Numerical results}

In this section, we have selected three planes which cut the rotary volume according to the three axes $\mathrm{x}=0 \mathrm{~mm}, \mathrm{y}=0$ $\mathrm{mm}$ and $\mathrm{z}=0 \mathrm{~mm}$ in order to visualize the following parameters: the static pressure, the average velocity, the turbulent kinetic energy, the dissipation rate of the turbulent kinetic energy and the turbulent viscosity.

\section{IV.1. Static pressure}

Figure 3 shows the distribution of the static pressure according to the chosen planes defined by $x=0 \mathrm{~mm}, \mathrm{y}=0$ $\mathrm{mm}$ and $\mathrm{z}=0 \mathrm{~mm}$. According to these results, it has been noted a variation of the distribution of the static pressure surrounding the propeller. Also, it has been observed that the static pressure in the top of the propeller is lower than in the bottom. Far from the propeller, there is no variation of the static pressure.

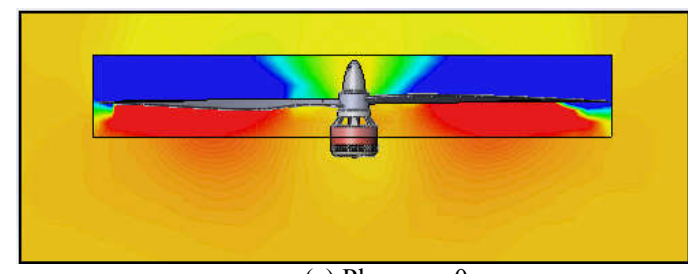

(a) Plane $x=0 \mathrm{~mm}$

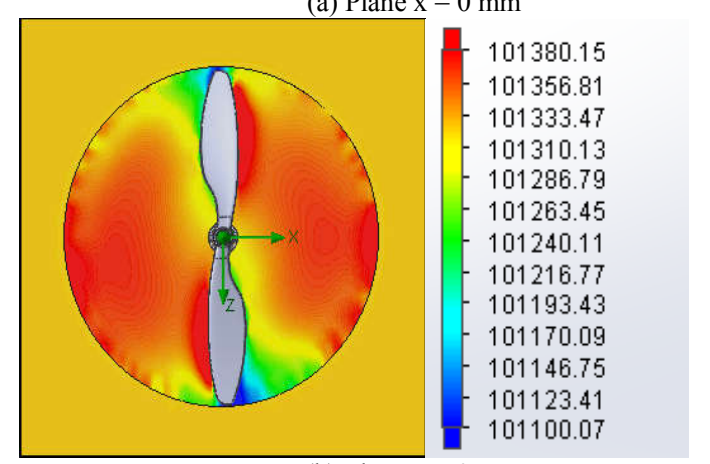

(b) Plane $\mathrm{y}=0 \mathrm{~mm}$

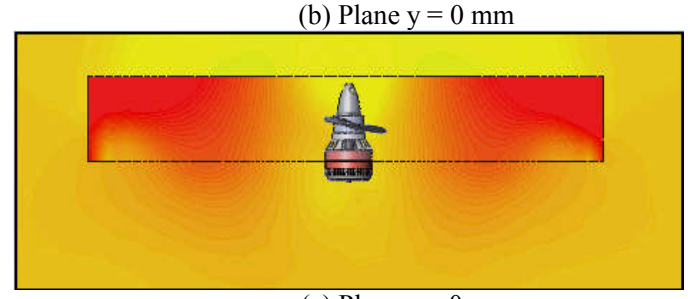

(c) Plane $z=0 \mathrm{~mm}$

Figure 3. Distribution of the static pressure

\section{IV.2. Average velocity}

Figure 4 presents the distribution of the average velocity in the planes defined by $x=0 \mathrm{~mm}, y=0 \mathrm{~mm}$ and $\mathrm{z}=0 \mathrm{~mm}$. According to these results, it has been noted that the important values of the average velocity are located in the bottom of the propeller. Far from the propeller, the velocity becomes lower.

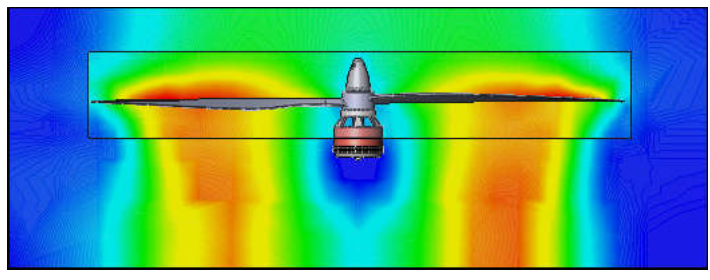

(a) Plane $x=0 \mathrm{~mm}$

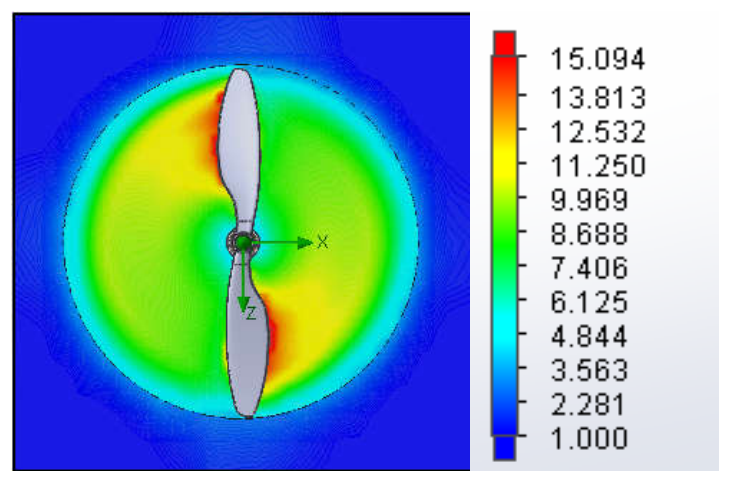

(b) Plane $y=0 \mathrm{~mm}$

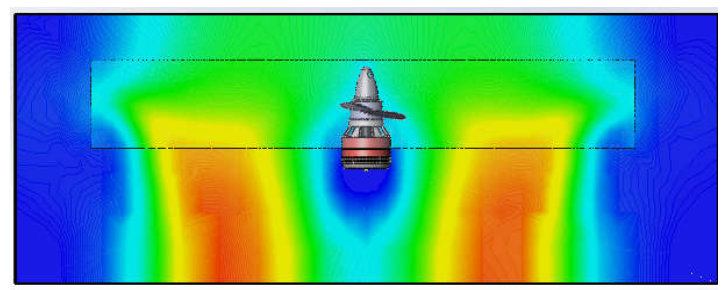

(c) Plane $\mathrm{z}=0 \mathrm{~mm}$

Figure 4. Distribution of the average velocity

\section{IV.3. Turbulent kinetic energy}

Figure 5 illustrates the distribution of the turbulent kinetic energy in different selected planes. When seeing the results, it has been noted that the wake zone characteristic of the important values of the turbulent kinetic energy is located at the extremities of the propeller as well as the extremities of the rotating volume. Due to the fact that the motor is fixed, there is a low variation of the turbulent kinetic energy at its neighbourhoods.

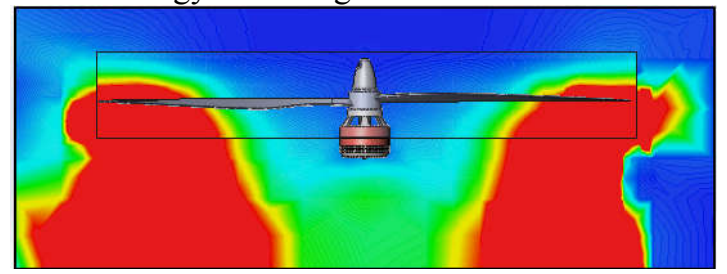

(a) Plane $\mathrm{x}=0 \mathrm{~mm}$ 


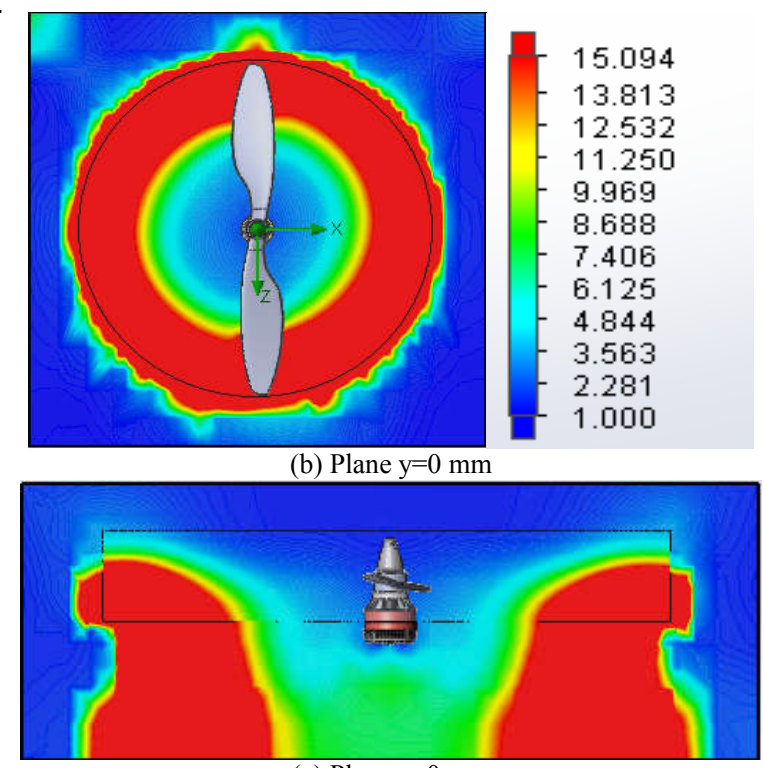

(c) Plane $\mathrm{z}=0 \mathrm{~mm}$

Figure 5. Distribution of the turbulent kinetic energy

\section{IV.4. Dissipation rate of the turbulent kinetic energy}

Figure 6 shows the distribution of the dissipation rate of the turbulent kinetic energy considered in the different planes defined by $\mathrm{x}=0 \mathrm{~mm}, \mathrm{y}=0 \mathrm{~mm}$ and $\mathrm{z}=0 \mathrm{~mm}$. As we see, the wake characteristic of the maximum values of the dissipation rate of the turbulent kinetic energy is concentrated at the extremities of the propeller as well as the extremities of the rotary volume. Near the motor, the values of the dissipation rate of the turbulent kinetic energy are low. This result is shown also at the exterior of the rotary volume. Indeed, it is clear that the zones where the turbulent kinetic energy is important, the dissipation rate of the turbulent kinetic energy is also high.

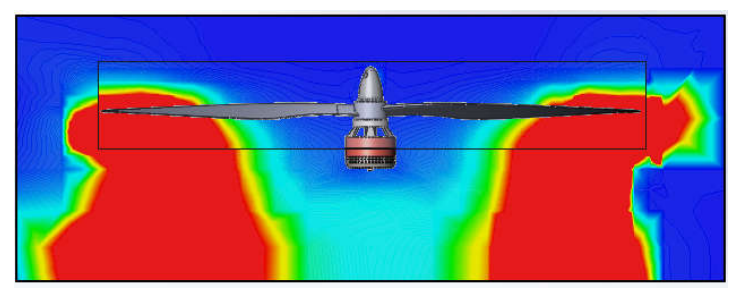

(a) Plane $\mathrm{x}=0 \mathrm{~mm}$
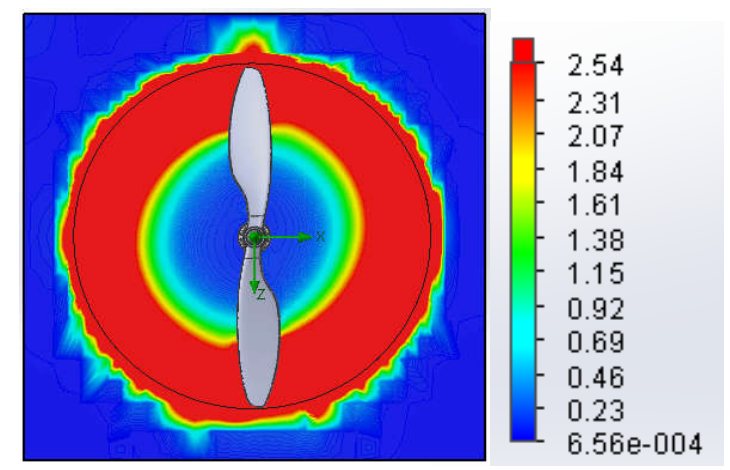

(b) Plane $\mathrm{y}=0 \mathrm{~mm}$

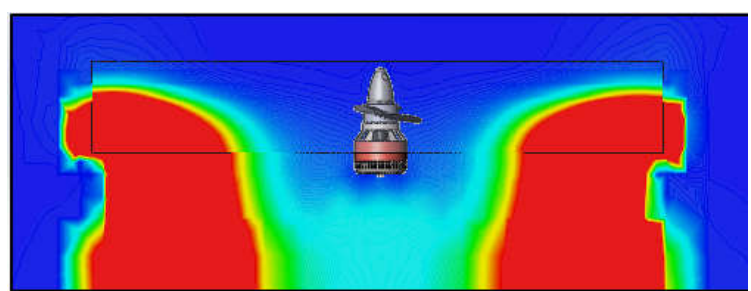

(c) Plane $\mathrm{z}=0 \mathrm{~mm}$

Figure 6. Distribution of the dissipation rate of the turbulent kinetic energy

\section{VI.5. Turbulent viscosity}

Figure 4.16 presents the distribution of the turbulent viscosity in the three different planes defined by $\mathrm{x}=0 \mathrm{~mm}$, $\mathrm{y}=0 \mathrm{~mm}$ and $\mathrm{z}=0 \mathrm{~mm}$. According to these results, it has been noted that the turbulent viscosity is important at the bottom of the propeller especially at its extremities. Indeed, it is clear that the turbulent viscosity presents a moderate or a low value, more precisely near the motor and in the outer of the rotating volume.

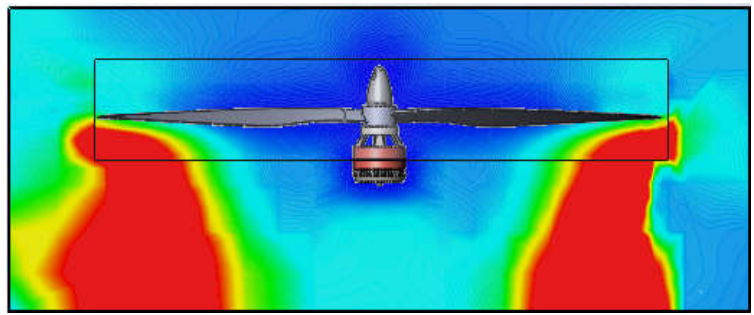

(a) Plane $\mathrm{x}=0 \mathrm{~mm}$

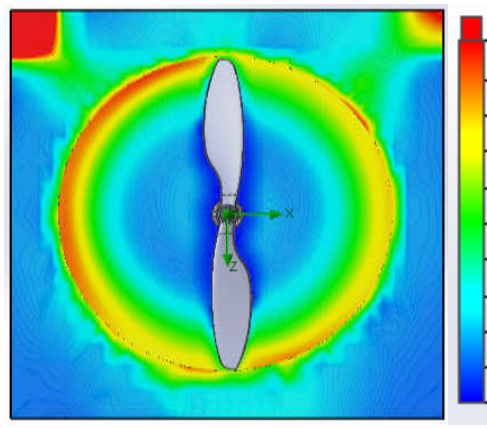

0.0001

$9.0340 \mathrm{e}-005$

$8.0302 \mathrm{e}-005$

$7.0265 \mathrm{e}-005$

$6.0227 \mathrm{e}-005$

$5.0190 \mathrm{e}-005$

$4.0153 \mathrm{e}-005$

$3.0115 \mathrm{e}-005$

$2.0078 \mathrm{e}-005$

$1.0041 \mathrm{e}-005$

(b) Plane $\mathrm{y}=0 \mathrm{~mm}$

$3.2524 \mathrm{e}-009$

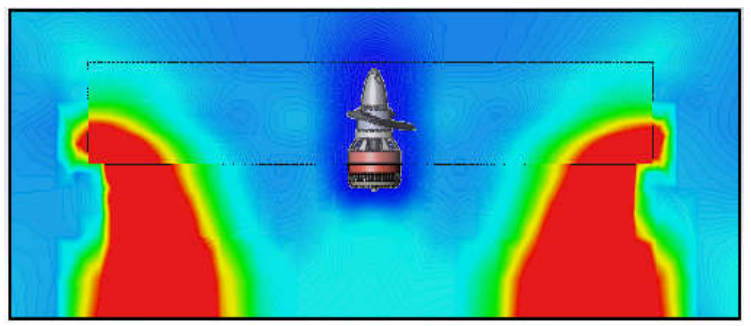

(c) Plane $\mathrm{z}=0 \mathrm{~mm}$

Figure 7. Distribution of the turbulent viscosity 


\section{Conclusion}

Computer simulations have been developed to study the aerodynamic structure around a NACA 6409 airfoil rotor. It has been noted that the size of the propeller as well as the type of the airfoil has a direct influence on the local characteristics of the aerodynamic structure. Particularly, it has been noted that the depression zones are located especially on the top of the propeller. Also, the wake zones are located at the extremities of the propeller and the rotating volume.

In further works, it will be interesting to determine the local characteristics around other airfoils as well as the curve of thrust in function of the rotational speed to discover the difference between them and deduce the airfoil that consumes less energy.

\section{Reference}

[1] D. Alexandrov, Light weight multicopter structural design for energy saving, Tallin University of technology, 2013.

[2] A. Le Pape, P. Beaumier, Numerical optimization of helicopter rotor aerodynamic performance in hover, Aerospace Science and Technology 9 (2005), pp. 191-201.

[3] X. Heyong, Y. Zhengyin, Numerical simulation of unsteady flow around forward flight helicopter with coaxial rotors, Chinese Journal of Aeronautics 24 (2011), pp. 1-7.

[4] J. Zhao, Q. Hou, H .Jin , Y.Zhu, G.Li, CFD analysis of ducted-fan UAV based on Magnus Effect, International Conference on Mechatronics and Automation, August 5-8, Chengdu China, Proceedings of 2012 IEEE.

[5] A. Brocklehurst, G. N.Barakos, A review of helicopter rotor blade tip shapes, Progress in aerospace sciences 56, (2013), pp. 35-74.

[6] Z. Driss, O. Mlayah, S. Driss, D. Driss, M. Maaloul, M.S. Abid, Study of the bucket design effect on the turbulent flow around unconventional Savonius wind rotors, Energy, Vol. 89, 2015. pp. 708-729.

[7] S. Driss, Z. Driss, I. Kallel Kammoun, Computational study and experimental validation of the heat ventilation in a living room with a solar patio system, Energy \& Buildings, Vol. 119, 2016, pp. 28-40.

[8] A. Bouabidi, Z. Driss, N. Cherif, M.S. Abid, Computational investigation of the external excitation frequency effect on liquid sloshing phenomenon, WSEAS Transactions on Fluid Mechanics, Vol 11, 2016, pp. 1-9.

[9] Z. Driss, O. Mlayah, S. Driss, M. Maaloul, M.S. Abid, Study of the incidence angle effect on the aerodynamic structure characteristics of an incurved Savonius wind rotor placed in a wind tunnel, Energy, Vol. 113, 2016, pp. 894-908. 